\title{
A Neutrosophic-Based Approach in Data Envelopment Analysis with Undesirable Outputs
}

\author{
Xinna Mao, ${ }^{1}$ Zhao Guoxi $\mathbb{D},{ }^{1}$ Mohammad Fallah, ${ }^{2}$ and S. A. Edalatpanah $\mathbb{D}^{3}$ \\ ${ }^{1}$ Department of Mathematics and Information Science, Xinxiang University, Xinxiang 453003, China \\ ${ }^{2}$ Department of Industrial Engineering, Islamic Azad University, Central Tehran Branch, Tehran, Iran \\ ${ }^{3}$ Department of Applied Mathematics, Ayandegan Institute of Higher Education, Tonekabon, Iran
}

Correspondence should be addressed to S. A. Edalatpanah; saedalatpanah@gmail.com

Received 7 April 2020; Revised 26 May 2020; Accepted 3 June 2020; Published 13 July 2020

Academic Editor: Harish Garg

Copyright (c) 2020 Xinna Mao et al. This is an open access article distributed under the Creative Commons Attribution License, which permits unrestricted use, distribution, and reproduction in any medium, provided the original work is properly cited.

Data Envelopment Analysis is one of the paramount mathematical methods to compute the general performance of organizations, which utilizes similar sources to produce similar outputs. Original DEA schemes involve crisp information of inputs and outputs that may not always be accessible in real-world applications. Nevertheless, in some cases, the values of the data are information with indeterminacy, impreciseness, vagueness, inconsistent, and incompleteness. Furthermore, the conventional DEA models have been originally formulated solely for desirable outputs. However, undesirable outputs may additionally be present in the manufacturing system, which wishes to be minimized. To tackle the mentioned issues and in order to obtain a reliable measurement that keeps original advantage of DEA and considers the influence of undesirable factors under the indeterminate environments, this paper presents a neutrosophic DEA model with undesirable outputs. The recommended technique is based on the aggregation operator and has a simple construction. Finally, an example is given to illustrate the new model and ranking approach in details.

\section{Introduction}

All organizations, whether governmental or private, require an effective performance assessment for development, growth, and sustainability in the competitive world of today so that within this growth it can appraise the efficiency and effectiveness of its organizational programs and its human resources processes. In other words, senior executive managers have always been seeking for a solution to ensure that their strategies are executed and, hence, have selected performance assessment methods as tools to implement their strategies. We should be mindful that novel measurement systems have been developed to target the existing strategies, and in this system, the indexes could be viewed as a crucial factor, in the present and future successes of the company. If these factors are alleviated or improved, then the company has launched its strategy.

The Data Envelopment Analysis (DEA) is a nonparametric method to analyse and assess the performance of decision-making units (DMUs), which converts several inputs into several outputs and considers the qualitative and quantitative criterions. Based on the work of [1], Charnes et al. [2] proposed this methodology that is called the CCR model. After that, Banker et al. [3] extended this model and established a new model to measure efficiency that is the socalled BBC model. With DEA, directors can achieve the relative efficiency of a set of DMUs. Note that the production function does not need to be known in this technique. In the recent years, an extensive application of DEA in numerous fields, such as banking institutions [4], insurance industry [5], financial services [6], education [7], sustainability [8], energy [9], and health-care services [10], has been observed. Furthermore, in the past four decades, numerous reports and articles have been published in esteemed global journals verifying that this method is operational, see [11-16].

However, in some cases, the values of the data are often information with indeterminacy, impreciseness, vagueness, inconsistent, and incompleteness. Inaccurate assessments 
are mainly the outcome of information that is unquantifiable, incomplete, and unavailable. By fuzzy sets [17], it is possible to model the uncertainty in information. In addition, there are several models of DEA with the fuzzy set, see [17-19] and references therein. However, fuzzy sets (FSs) deliberate only the membership function and cannot arrange other parameters of vagueness. Therefore, intuitionistic fuzzy sets (IFSs) have been introduced in [20], see also the Pythagorean fuzzy sets [21-23].

Though IFSs is able to address incomplete data for numerous real-world topics, it cannot handle other natures of uncertainty such as indeterminate information. Hence, Smarandache [24] puts forward an indeterminacy degree of membership as an autonomous element and proposes the neutrosophic set (NS). Then, inspired by sports (winner, loser, and equal), voting (agree, disagree, and abstain), answering (yes, no, and I do not know), decision-making (decision, no decision, and doubt), and acceptance (accept, rejection, and suspension) and since the principle of excluded middle cannot be applied to new logic, he combines three-valued logic with nonstandard analysis. Since then, a series of subclasses of NS have emerged, mainly including interval neutrosophic set [25-27], bipolar neutrosophic set $[28,29]$, single-valued neutrosophic set [30-33], simplified neutrosophic sets [34-36], neutrosophic structured element [37], multivalued neutrosophic set [38, 39], and neutrosophic linguistic set $[40,41]$ which have been proposed. Moreover, these concepts utilized in several problems, see [42-51].

In the DEA literature, there are also some models of DEA with IFSs, see [52-60]. However, in actual activities, there are some indeterminate information. If the indeterminacy factors are ignored, the relative effectiveness of DMUs will still be evaluated using the DEA model established on the basis of determinate values and biased or even wrong information will be obtained, which will bring some errors to management decisions. Therefore, it seems convenient and necessary to consider the neutrosophic DEA model.

As far as we know, there are few studies concerning DEA with neutrosophic information. The utilization of neutrosophic set in DEA can be traced to Edalatpanah [61] and additional investigations have been accessible in [62-67]. However, these neutrosophic DEA methods are formulated solely for desirable outputs and cannot eliminate the influence of undesirable factors on the efficiency evaluation. We know that the undesirable outputs may additionally be present in the manufacturing system that needs to be minimized. For example, in banking industry nonperforming loans/assets and in manufacturing systems, the production of a variety of emissions, pollutions, and industrial waste gas are undesirable outputs [68-73]. Therefore, there is still a need from the neutrosophic DEA method to develop a new model that keeps original advantage and considers the influence of undesirable factors.
Consequently, in this study, we establish a novel method of DEA with undesirable outputs in which all data are singlevalued neutrosophic sets (SVNSs). Furthermore, a competent algorithm for solving the new DEA model has been presented. The main contributions of this paper are four folds: (1) we model the indeterminacies in the input and output data using SVNSs; (2) the proposed approach considers the impact of undesirable outputs on the performance of DMUs; (3) we provide a theorem regarding the feasibility and boundedness of the solution of new model; (4) we determine the efficiency scores of the DMUs as crisp values.

The paper unfolds as follows. Some basic knowledge and concepts on DEA and its models are deliberated in Section 2. In Section 3, some knowledge and arithmetic operations on neutrosophic sets are discussed. In Section 4, we propose a new model of neutrosophic DEA with undesirable outputs and establish an algorithm to solve it. In Section 5, the proposed model and the related algorithm are illustrated with a numerical example to ensure their validity and usefulness over the existing models. Finally, conclusions and future direction are offered in Section 6.

\section{The Basic Concepts of DEA and its Models}

DEA is a mathematical programming methodology that allows performance measurement of homogeneous DMUs that have several inputs and outputs. In DEA approach, there is no need to determine the specific form of the production function, and linear programming is needed to construct a piecewise linear surface (frontier) to cover all data, and then the efficiency of each of the DMUs is calculated from this frontier. The frontier obtained is the efficiency boundary, where the points on it are efficient, and other DMUs that are inside the cover surface are inefficient.

Let DMUO be under consideration; then, the production possibility set of the BCC model proposed by Banker et al. [3] is as follows:

$$
\begin{aligned}
T_{\mathrm{BCC}}= & \left\{(x, y) \mid \sum_{q=1}^{n} \alpha_{q} x_{q} \leq x, \sum_{q=1}^{n} \alpha_{q} y_{q} \geq y, \sum_{q=1}^{n} \alpha_{q}=1,\right. \\
& \left.\alpha_{q} \geq 0, q=1, \ldots, n\right\} .
\end{aligned}
$$

Therefore, the BCC model is as follows:

$$
\begin{array}{ll}
\text { Min } & \eta_{o} \\
& \sum_{q=1}^{n} \alpha_{q} x_{p q} \leq \eta_{o} x_{p_{o}}, \quad p=1, \ldots, m \\
\text { s.t. } & \sum_{q=1}^{n} \alpha_{j} y_{s q} \geq y_{s o}, \quad s=1, \ldots, k \\
& \sum_{q=1}^{n} \alpha_{q}=1, \quad \alpha_{q} \geq 0, q=1, \ldots, n .
\end{array}
$$


For the BCC model with undesirable outputs, Guo and $\mathrm{Wu}$ [68] proposed

$$
\begin{array}{ll}
\text { Min } \quad & \eta_{o} \\
& \sum_{q=1}^{n} \alpha_{q} x_{p q} \leq \eta_{o} x_{p_{o}}, \quad p=1, \ldots, m \\
& \\
\text { s.t } \quad & \sum_{q=1}^{n} \alpha_{j} y_{s q}^{g} \geq y_{s o}^{g}, \quad s=1, \ldots, k \\
& \sum_{q=1}^{n} \alpha_{j} y_{s^{\prime} q}^{b} \leq \eta_{o} y_{s^{\prime} o}^{b}, \quad s^{\prime}=1, \ldots, k \\
& \sum_{q=1}^{n} \alpha_{q}=1, \quad \alpha_{q} \geq 0, q=1, \ldots, n .
\end{array}
$$

In Model (3), we assume that there are $n$ DMUs that use inputs $x_{p q}(p=1, \ldots, m)$, to achieve desirable outputs (good) $y_{s q}^{g}(s=1, \ldots, k)$ and undesirable outputs (bad) $y_{s^{\prime} q}^{b}\left(s^{\prime}=1, \ldots, k^{\prime}\right)$ After calculation, if $\eta_{o}=1$, then $\mathrm{DMU}_{\mathrm{o}}$ is efficient; else, it is inefficient.

\section{Neutrosophic Sets}

Smarandache has given a number of actual examples for potential applications of NS; nevertheless, usage of NSs in real applied problems is difficult. Hence, NSs reduced into a type of SVNSs that will preserve the processes of the NSs. Here, we will discuss some basic definitions related to neutrosophic sets and SVNSs, respectively [30-33].

Definition 1. In universal U, a NS is distinct with truth, falsity, and indeterminacy membership functions of $x$ in the real nonstandard $]^{-} 0,1^{+}$, where the summation of them belong to $[0,3]$. If these functions are singleton in the $[0,1]$, then a SVNS $\psi$ is denoted by

$$
\psi=\left\{\left(x, \hbar_{\psi}(x), \ell_{\psi}(x), \lambda_{\psi}(x)\right) \mid x \in U\right\} .
$$

Furthermore, $\psi=\left\langle\hbar_{\psi}, \ell_{\psi}, \lambda_{\psi}\right\rangle$ is called the single-valued neutrosophic number (SVNN) if $U$ has just one component.

Definition 2. Suppose that $\mathrm{C}$ and $\mathrm{D}$ be two SVNSs, then $\mathrm{C} \subseteq$ $\mathrm{D}$ if and only if $\hbar_{C}(x) \leq \hbar_{D}(x), \ell_{C}(x) \geq \ell_{D}(x)$, and $\lambda_{C}(x) \geq \lambda_{D}(x)$.

Definition 3. LetMandNbe two SVNNs. Then, equations (5) to $(8)$ are true:

$$
\begin{aligned}
\text { (i) } M \oplus N= & \left\langle\hbar_{M}+\hbar_{N}-\hbar_{M} \hbar_{N}, \ell_{M} \ell_{N}, \lambda_{M} \lambda_{N}\right\rangle, \\
\text { (ii) } M \otimes N= & \left\langle\hbar_{M} \hbar_{N}, \ell_{M}+\ell_{N}-\ell_{M} \ell_{N}, \lambda_{M}\right. \\
& \left.+\hbar_{N}-\lambda_{M} \lambda_{N}\right\rangle,
\end{aligned}
$$

(iii) $\alpha \mathrm{M}=\left\langle 1-\left(1-t \hbar_{\mathrm{M}} n(x)\right)^{\alpha},\left(\ell_{\mathrm{M}}(x)\right)^{\alpha}\right.$,

$$
\left.\cdot\left(\lambda_{\mathrm{M}}(x)\right)^{\alpha}\right\rangle, \quad \alpha>0 \text {, }
$$

(iv) $\mathrm{M}^{\alpha}=\left\langle\left(\hbar_{\mathrm{M}}(x)\right)^{\alpha}, 1-\left(1-\ell_{\mathrm{M}}(x)\right)^{\alpha}\right.$,

$$
\left.1-\left(1-\lambda_{M}(x)\right)^{\alpha}\right\rangle, \quad \alpha>0 \text {. }
$$

Definition 4. Suppose that $\mathrm{M}_{q}=\left\langle\hbar_{\mathrm{M}_{q}}, \ell_{\mathrm{M}_{q}}, \lambda_{\mathrm{M}_{q}}\right\rangle$ $(q=1, \ldots, n)$ be a collection SVNNs. Then, the related weighted arithmetic average operator is

$$
F_{W}\left(\mathrm{M}_{1}, \ldots, \mathrm{M}_{n}\right)=\sum_{q=1}^{n} \omega_{q} \mathrm{M}_{q}
$$

and the aggregation operator is defined as follows:

$$
F_{W}\left(\mathrm{M}_{1}, \ldots, \mathrm{M}_{n}\right)=\left\langle 1-\prod_{q=1}^{n}\left(1-\hbar_{\mathrm{M}_{q}}(x)\right)^{\omega_{q}}, \prod_{q=1}^{n}\left(\ell_{\mathrm{M}_{q}}(x)\right)^{\omega_{q}}, \prod_{q=1}^{n}\left(\lambda_{\mathrm{M}_{q}}(x)\right)^{\omega_{q}}\right\rangle
$$

where $W=\left(\omega_{1}, \omega_{2}, \ldots, \omega_{n}\right)$ is the weight vector of $\mathrm{M}_{q}, \omega_{q} \in[0,1]$ and $\sum_{q=1}^{n} \omega_{q}=1$.

\section{Proposed Model}

In the DEA literature, there are some models of DEA with neutrosophic information, see [61-67]. Nevertheless, these models work solely for desirable outputs and cannot consider undesirable outputs. Hence, there is a requisite to develop a new model that keeps original advantage of neutrosophic DEA models and considers the influence of undesirable outputs. In this section, we propose an inputoriented BCC model with undesirable outputs in which all data are SVNNs. Furthermore, we propose an algorithm for solving the new DEA model. Moreover, a theorem regarding the feasibility and boundedness of the solution of the new model has been provided. For the qth DMU, consider the input $\tilde{x}_{p q}=\left\langle\hbar_{x_{p q}}, \ell_{x_{p q}}, \lambda_{x_{p q}}\right\rangle$, desirable outputs $\tilde{y}_{s q}^{g}=\left\langle\hbar_{y_{s q}^{g}}, \ell_{y_{s q}^{g}}, \lambda_{y_{s q}^{g}}\right\rangle$, and undesirable outputs $\tilde{y}_{s q}^{b}=\left\langle\hbar_{y_{s q}^{b}}, \ell_{y_{s q}^{b}}, \lambda_{y_{s q}^{b}}\right\rangle$ which are the SVNNs. Thus, by combinations of the BCC model under the single-valued neutrosophic environment with undesirable outputs, we have the following model:

$$
\begin{array}{ll}
\operatorname{Min} & \eta_{o} \\
& \sum_{q=1}^{n} \alpha_{q} \tilde{x}_{p q} \leq \eta_{o} \tilde{x}_{p_{o}}, \quad p=1, \ldots, m \\
& \sum_{q=1}^{n} \alpha_{j} \tilde{y}_{s q}^{g} \geq \tilde{y}_{s o}^{g}, \quad s=1, \ldots, k \\
& \sum_{q=1}^{n} \alpha_{j} \tilde{y}_{s^{\prime} q}^{b} \leq \eta_{o} \tilde{y}_{s^{\prime} o}^{b}, \quad s^{\prime}=1, \ldots, k^{\prime} \\
& \sum_{q=1}^{n} \alpha_{q}=1, \quad \alpha_{q} \geq 0, q=1, \ldots, n .
\end{array}
$$


Step 1. Construct the problem based on Model (10).

Step 2. According to Definitions 3 and 4, transform the SVNBCC-UO model of Step 1 into

Min $\eta_{0}$

$$
\begin{aligned}
& \eta_{o} \\
& \left\langle 1-\prod_{q=1}^{n}\left(1-\hbar_{x_{p q}}\right)^{\alpha_{q}}, \prod_{q=1}^{n}\left(\ell_{x_{p q}}\right)^{\alpha_{q}}, \prod_{q=1}^{n}\left(\lambda_{x_{p q}}\right)^{\alpha_{q}}\right\rangle \leq\left\langle 1-\left(1-\hbar_{x_{p o}}\right)^{\eta_{o}},\left(\ell_{x_{p o}}\right)^{\eta_{o}},\left(\lambda_{x_{p o}}\right)^{\eta_{o}}\right\rangle, \quad p=1, \ldots, m \\
& \left\langle 1-\prod_{q=1}^{n}\left(1-\hbar_{y_{s q}^{g}}\right)^{\alpha_{q}}, \prod_{q=1}^{n}\left(\ell_{y_{s q}^{g}}\right)^{\alpha_{q}}, \prod_{q=1}^{n}\left(\lambda_{y_{s q}^{g}}\right)^{\alpha_{q}}\right\rangle \geq\left\langle\hbar_{y_{s o}^{g}}, \ell_{y_{s o}^{g}}, \lambda_{y_{s o}^{g}}\right\rangle, \quad s=1, \ldots, k \\
& \left\langle 1-\prod_{q=1}^{n}\left(1-\hbar_{y_{s^{\prime} q}^{b}}\right)^{\alpha_{q}}, \prod_{q=1}^{n}\left(\ell_{y_{s^{\prime} q}^{b}}\right)^{\alpha_{q}}, \prod_{q=1}^{n}\left(\lambda_{y_{s^{\prime} q}^{b}}\right)^{\alpha_{q}}\right\rangle \leq\left\langle 1-\left(1-\hbar_{y_{s^{\prime}}^{b}}\right)^{\eta_{o}},\left(\ell_{y_{s^{\prime}}^{b}}\right)^{\eta_{o}},\left(\lambda_{y_{s^{\prime}}^{b}}\right)^{\eta_{o}}\right\rangle, \quad s^{\prime}=1, \ldots, k^{\prime} \\
& \sum_{q=1}^{n} \alpha_{q}=1 \\
& \alpha_{q} \geq 0, \quad q=1, \ldots, n .
\end{aligned}
$$

S.t.

Step 3. By Definition 2, transform Step 2 into

Min $\eta_{0}$

$\prod_{q=1}^{n}\left(1-\hbar_{x_{p q}}\right)^{\alpha_{q}} \geq\left(1-\hbar_{x_{p o}}\right)^{\eta_{o}}, \quad p=1,2, \ldots, m$

$\prod_{q=1}^{n}\left(\ell_{x_{p q}}\right)^{\alpha_{q}} \geq\left(\ell_{x_{p o}}\right)^{\eta_{o}}, \quad p=1,2, \ldots, m$

$\prod_{q=1}^{n}\left(\lambda_{x_{p q}}\right)^{\alpha_{q}} \geq\left(\lambda_{x_{p o}}\right)^{\eta_{o}}, \quad p=1,2, \ldots m$

$\prod_{q=1}^{n}\left(1-\hbar_{y_{s q}^{g}}\right)^{\alpha} \leq\left(1-\hbar_{y_{s o}^{g}}\right), \quad s=1, \ldots, k$

$\prod_{q=1}^{n}\left(\ell_{y_{s q}^{g}}\right)^{\alpha_{q}} \leq \ell_{y_{s o}^{g}}, \quad s=1, \ldots, k$

s.t.

$$
\begin{aligned}
& \prod_{q=1}^{n}\left(\lambda_{y_{s q}^{g}}\right)^{\alpha_{q}} \leq \lambda_{y_{s o}^{g}}, \quad s=1, \ldots, k \\
& \prod_{q=1}^{n}\left(1-\hbar_{y^{b}{ }^{b}}\right)^{\alpha_{q}} \geq\left(1-\hbar_{y_{s^{\prime}}{ }^{\prime}}\right)^{\eta_{o}}, \quad s^{\prime}=1, \ldots, k^{\prime} \\
& \prod_{q=1}^{n}\left(\ell_{y_{s^{\prime} q}^{b}}\right)^{\alpha_{q}} \geq\left(\ell_{y_{s^{\prime} o}}\right)^{\eta_{o}}, \quad s^{\prime}=1, \ldots, k^{\prime} \\
& \prod_{q=1}^{n}\left(\lambda_{y_{s^{\prime},}^{b}}\right)^{\alpha_{q}} \geq\left(\lambda_{y_{s^{\prime}}^{b}}\right)^{\eta_{o}}, \quad s^{\prime}=1, \ldots, k^{\prime} \\
& \sum_{q=1}^{n} \alpha_{q}=1 \text {, } \\
& \alpha_{q} \geq 0, \quad q=1, \ldots, n \text {. }
\end{aligned}
$$

Step 4. Convert Step 3 into the following linear model:

$$
\begin{aligned}
\text { Min } & \eta_{o} \\
& \sum_{q=1}^{n} \alpha_{q} \ln \left(1-\hbar_{x_{p q}}\right) \geq \eta_{o} \ln \left(1-\hbar_{x_{p o}}\right), \quad p=1,2, \ldots, m \\
& \sum_{q=1}^{n} \alpha_{q} \ln \left(\ell_{x_{p q}}\right) \geq \eta_{o} \ln \left(\ell_{x_{p o}}\right), \quad p=1,2, \ldots, m \\
& \sum_{q=1}^{n} \alpha_{q} \ln \left(\lambda_{x_{p q}}\right) \geq \eta_{o} \ln \left(\lambda_{x_{p o}}\right), \quad p=1,2, \ldots, m \\
& \sum_{q=1}^{n} \alpha_{q} \ln \left(1-\hbar_{y_{s q}^{g}}\right) \leq \ln \left(1-\hbar_{y_{s o}^{g}}\right), \quad s=1, \ldots, k \\
& \sum_{q=1}^{n} \alpha_{q} \ln \left(\ell_{y_{s q}^{g}}\right) \leq \ln \left(\ell_{y_{s o}^{g}}\right), \quad s=1, \ldots, k \\
\text { s.t } \quad & \sum_{q=1}^{n} \alpha_{q} \ln \left(\lambda_{y_{s q}^{g}}\right) \leq \ln \left(\lambda_{y_{s o}^{g}}\right), \quad s=1, \ldots, k \\
& \sum_{q=1}^{n} \alpha_{j} \ln \left(1-\hbar_{y_{s^{\prime} q}^{b}}\right) \geq \eta_{o} \ln \left(1-\hbar_{y_{s^{\prime}}^{b}}\right), \quad s^{\prime}=1, \ldots, k^{\prime} \\
& \sum_{q=1}^{n} \alpha_{j} \ln \left(\ell_{y_{s^{\prime} q}^{b}}\right) \geq \eta_{o} \ln \left(\ell_{y_{s^{\prime} o}^{b}}\right), \quad s^{\prime}=1, \ldots, k^{\prime} \\
& \sum_{q=1}^{n} \alpha_{j} \ln \left(\lambda_{y_{s^{\prime}}^{b}}\right) \geq \eta_{o} \ln \left(\lambda_{y_{s^{\prime} o}^{b}}\right), \quad s^{\prime}=1, \ldots, k^{\prime} \\
& \sum_{q=1}^{n} \alpha_{q}=1 \\
& \alpha_{q} \geq 0, \quad q=1, \ldots, n .
\end{aligned}
$$

Step 5. Using Step 4, get the optimal efficiency of each DMU.

Algorithm 1: Solution of the SVNBCC-UO model.

We called this model as single-valued neutrosophic BCC with undesirable outputs (SVNBCC-UO). Now, we present an algorithm to solve Model (11).

In the following, we show that our model is feasible and bounded.

Theorem 1. The model of SVNBCC-UO is feasible and bounded. Furthermore, its optimal objective function is 1.
Proof. From Algorithm 1, we can transform the model SVNBCC-UO into Step 4 in Algorithm 1. So, with the solution $\alpha_{q}=\left\{\begin{array}{ll}1, & q=o, \\ 0, & \text { else, }\end{array}\right.$ and $\eta_{o}=1$, it is easy to see that the Step 4 in Algorithm 1 is always feasible. Thus, regardless of the values of inputs and outputs, there is always at least one feasible solution for Step 4 in Algorithm 1. Because the above solution is feasible along with the objective function of Step 4 
TABLE 1: Input information of the 15 DMUs.

\begin{tabular}{lcrr}
\hline DMUS & Input 1 & Input 2 & Input 3 \\
\hline 1 & $\langle 0.6,0.4,0.3\rangle$ & $\langle 0.5,0.4,0.3\rangle$ & $\langle 0.7,0.3,0.4\rangle$ \\
2 & $\langle 0.3,0.1,0.2\rangle$ & $\langle 0.6,0.5,0.1\rangle$ & $\langle 0.6,0.4,0.5\rangle$ \\
3 & $\langle 0.4,0.2,0.1\rangle$ & $\langle 0.2,0.6,0.3\rangle$ & $\langle 0.8,0.2,0.1\rangle$ \\
4 & $\langle 0.5,0.5,0.2\rangle$ & $\langle 0.2,0.3,0.1\rangle$ & $\langle 0.5,0.2,0.2\rangle$ \\
5 & $\langle 0.3,0.2,0.4\rangle$ & $\langle 0.2,0.8,0.7\rangle$ & $\langle 0.7,0.2,0.7\rangle$ \\
6 & $\langle 0.5,0.2,0.2\rangle$ & $\langle 0.7,0.5,0.1\rangle$ & $\langle 0.7,0.3,0.2\rangle$ \\
7 & $\langle 0.7,0.1,0.2\rangle$ & $\langle 0.4,0.4,0.2\rangle$ & $\langle 0.7,0.3,0.2\rangle$ \\
8 & $\langle 0.2,0.6,0.5\rangle$ & $\langle 0.1,0.7,0.2\rangle$ & $\langle 0.3,0.9,0.6\rangle$ \\
9 & $\langle 0.6,0.3,0.3\rangle$ & $\langle 0.5,0.2,0.5\rangle$ & $\langle 0.9,0.2,0.3\rangle$ \\
10 & $\langle 0.4,0.7,0.7\rangle$ & $\langle 0.8,0.2,0.6\rangle$ & $\langle 0.6,0.2,0.3\rangle$ \\
11 & $\langle 0.7,0.3,0.3\rangle$ & $\langle 0.4,0.1,0.3\rangle$ & $\langle 0.5,0.1,0.4\rangle$ \\
12 & $\langle 0.6,0.3,0.5\rangle$ & $\langle 0.5,0.5,0.3\rangle$ & $\langle 0.6,0 ., 0.3\rangle$ \\
13 & $\langle 0.5,0.2,0.4\rangle$ & $\langle 0.9,0.3,0.3\rangle$ & $\langle 0.8,0.1,0.4\rangle$ \\
14 & $\langle 0.4,0.3,0.1\rangle$ & $\langle 0.8,0.4,0.1\rangle$ & $\langle 0.9,0.2,0.3\rangle$ \\
15 & $\langle 0.6,0.4,0.3\rangle$ & $\langle 0.5,0.1,0.1\rangle$ & $\langle 0.6,0.1,0.1\rangle$ \\
\hline
\end{tabular}

TABLe 2: Output information of the 15 DMUs.

\begin{tabular}{lccc}
\hline DMUS & Output 1(good) & Output 2 (good) & Output 3 (bad) \\
\hline 1 & $\langle 0.5,0.5,0.7\rangle$ & $\langle 0.5,0.4,0.4\rangle$ & $\langle 0.5,0.2,0.1\rangle$ \\
2 & $\langle 0.2,0.7,0.5\rangle$ & $\langle 0.7,0.2,0.5\rangle$ & $\langle 0.4,0.1,0.1\rangle$ \\
3 & $\langle 0.3,0.5,0.8\rangle$ & $\langle 0.6,0.7,0.5\rangle$ & $\langle 0.6,0.2,0.1\rangle$ \\
4 & $\langle 0.2,0.5,0.7\rangle$ & $\langle 0.3,0.5,0.6\rangle$ & $\langle 0.5,0.2,0.1\rangle$ \\
5 & $\langle 0.8,0.4,0.3\rangle$ & $\langle 0.8,0.5,0.1\rangle$ & $\langle 0.3,0.1,0.6\rangle$ \\
6 & $\langle 0.3,0.6,0.7\rangle$ & $\langle 0.1,0.6,0.7\rangle$ & $\langle 0.8,0.1,0.2\rangle$ \\
7 & $\langle 0.5,0.2,0.6\rangle$ & $\langle 0.5,0.5,0.4\rangle$ & $\langle 0.9,0.2,0.3\rangle$ \\
8 & $\langle 0.3,0.3,0.6\rangle$ & $\langle 0.7,0.4,0.7\rangle$ & $\langle 0.3,0.3,0.3\rangle$ \\
9 & $\langle 0.6,0.4,0.4\rangle$ & $\langle 0.5,0.5,0.5\rangle$ & $\langle 0.4,0.2,0.1\rangle$ \\
10 & $\langle 0.2,0.3,0.7\rangle$ & $\langle 0.5,0.3,0.3\rangle$ & $\langle 0.5,0.4,0.1\rangle$ \\
11 & $\langle 0.3,0.8,0.5\rangle$ & $\langle 0.2,0.7,0.6\rangle$ & $\langle 0.5,0.2,0.2\rangle$ \\
12 & $\langle 0.6,0.1,0.4\rangle$ & $\langle 0.2,0.2,0.3\rangle$ & $\langle 0.1,0.5,0.5\rangle$ \\
13 & $\langle 0.4,0.7,0.8\rangle$ & $\langle 0.4,0.8,0.6\rangle$ & $\langle 0.7,0.1,0.1\rangle$ \\
14 & $\langle 0.5,0.5,0.6\rangle$ & $\langle 0.5,0.3,0.5\rangle$ & $\langle 0.5,0.2,0.4\rangle$ \\
15 & $\langle 0.2,0.4,0.8\rangle$ & $\langle 0.7,0.4,0.6\rangle$ & $\langle 0.4,0.1,0.1\rangle$ \\
\hline & & &
\end{tabular}

in Algorithm 1 is minimization, the best value regarding the objective function is certainly lower than or equal to 1 .

\section{Numerical Experiment}

In this section, the proposed SVNBCC-UO and the related algorithm are illustrated with a numerical example to ensure their validity and usefulness over the existing models.

Example 1. Consider an efficiency problem with 15 DMUs, three inputs, two desirable outputs, and one undesirable output that all information are presented as SVNNs (see Tables 1 and 2).

Here, we used Algorithm 1 for solving the SVNBCC-UO model. Suppose that $\mathrm{DMU}_{1}$ is under consideration, so based on Algorithm 1 we have the following.

First, we construct the model using Steps $1-3$. Then, by means of Step 4, we construct the Min $\eta_{1}$ with the constraints of Step 4 in Algorithm 1. For example, by Step 4 in Algorithm 1 for Input 1, we have the following relation:
TABle 3: The efficiencies of the DMUs.

\begin{tabular}{lcc}
\hline DMUS & Efficiency $\left(\eta^{*}\right)$ & Rank \\
\hline DMU1 & 0.8329 & 6 \\
DMU2 & 0.7504 & 8 \\
DMU3 & 0.9451 & 4 \\
DMU4 & 0.8197 & 7 \\
DMU5 & 1.0000 & $\mathbf{1}$ \\
DMU6 & 0.5822 & 12 \\
DMU7 & 0.7430 & 9 \\
DMU8 & 1.0000 & $\mathbf{1}$ \\
DMU9 & 0.9821 & 2 \\
DMU10 & 1.0000 & $\mathbf{1}$ \\
DMU11 & 0.9463 & 3 \\
DMU12 & 1.0000 & $\mathbf{1}$ \\
DMU13 & 0.7270 & 10 \\
DMU14 & 0.8987 & 5 \\
DMU15 & 0.6549 & 11 \\
\hline
\end{tabular}

$$
\begin{aligned}
& \alpha_{1} \ln (1-0.6)+\alpha_{2} \ln (1-0.3)+\alpha_{3} \ln (1-0.4)+\alpha_{4} \ln (1-0.5) \\
+ & \alpha_{5} \ln (1-0.3)+\alpha_{6} \ln (1-0.5)+\alpha_{7} \ln (1-0.7)+\alpha_{8} \ln (1-0.2) \\
+ & \alpha_{9} \ln (1-0.6)+\alpha_{10} \ln (1-0.4)+\alpha_{11} \ln (1-0.7)+\alpha_{12} \ln (1-0.6) \\
+ & \alpha_{13} \ln (1-0.5)+\alpha_{14} \ln (1-0.4)+\alpha_{15} \ln (1-0.6) \geq \eta_{1} \ln (1-0.6) .
\end{aligned}
$$

So, after calculations with Matlab, we get $\eta_{1}^{*}=0.9068$ for $D M U_{1}$. Correspondingly, the technical efficiencies of each $\mathrm{DMU}_{\mathrm{i}}$ are measured using the proposed algorithm and are presented in Table 3.

To validate the presented efficiencies, these efficiencies were compared with the efficiencies obtained by the existing neutrosophic DEA methods and are given in Figure 1. In this figure, we can see the impact of undesirable outputs on the performance of DMUs. From Figure 1, we can see that the efficiencies of DMUs are found to be smaller for SVNBCCUO compared to the existing neutrosophic DEA methods. It is interesting that DMUs $1-4,6-7$, and 9 are efficient in the existing neutrosophic DEA methods; however, they are actually inefficient with the efficiency scores of 0.8329 , $0.7504,0.9451,0.8197,0.5822,0.7430$, and 0.9821 using 


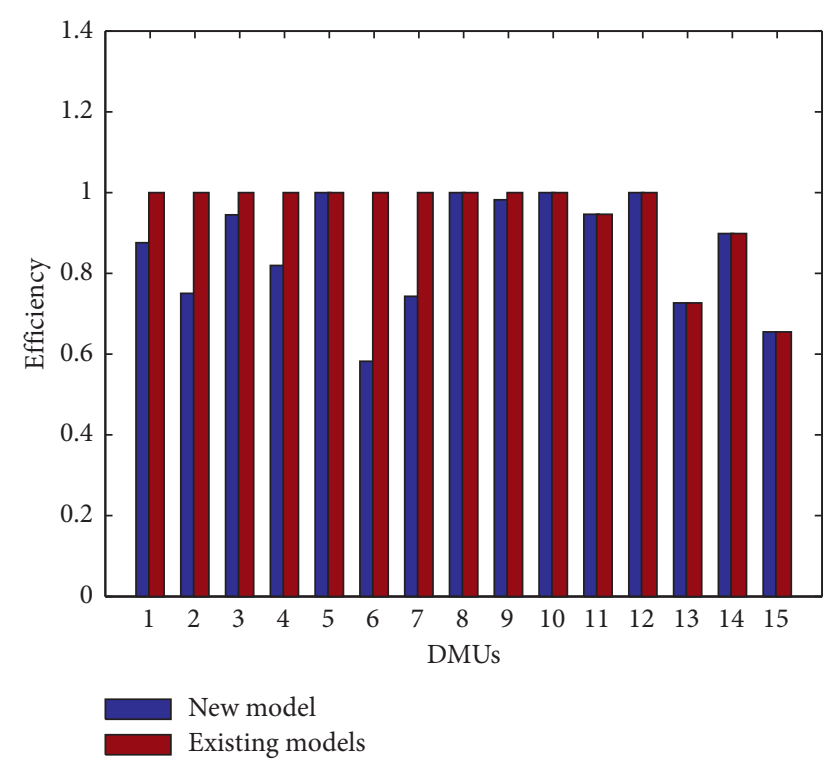

FIgURE 1: Comparison of the new model and the existing models.

SVNBCC-UO, respectively. Therefore, SVNBCC-UO is more realistic than the existing neutrosophic DEA methods. Therefore, if we solve the mentioned problem with the existing methods, we cannot get to a reliable evaluation.

\section{Conclusions and Future Work}

This paper, established a new strategy to solve a neutrosophic data envelopment analysis model with undesirable outputs. In comparison with the existing neutrosophic DEA methods, the significant characteristic of the new DEA method is that it can handle the undesirable outputs simply and effectively. A numerical experiment and comparison results with the existing models have been demonstrated to display the competence of the presented method. The proposed methodology has created hopeful consequences from computing and performance facets. It is worth mentioning that the uncertainty, ambiguity, and indeterminacy in this paper are limited to single-valued neutrosophic numbers. Nevertheless, the other types of numbers such as bipolar NSs and interval-valued neutrosophic numbers, Pythagorean fuzzy set, and $q$-rung orthopair fuzzy set can also be used to indicate variables characterizing the core in world-wide problems. As for future research, we intend to extend the proposed approach to these kinds of tools.

\section{Data Availability}

The data used to support the findings of this study are included within the article.

\section{Conflicts of Interest}

The authors declare no conflicts of interest.

\section{Acknowledgments}

This work was supported by Xinxiang University.

\section{References}

[1] M. J. Farrell, "The measurement of productive efficiency," Journal of the Royal Statistical Society. Series A (General), vol. 120, no. 3, p. 253, 1957.

[2] A. Charnes, W. W. Cooper, and E. Rhodes, "Measuring the efficiency of decision making units," European Journal of Operational Research, vol. 2, no. 6, pp. 429-444, 1978.

[3] R. D. Banker, A. Charnes, and W. W. Cooper, "Some models for estimating technical and scale inefficiencies in data envelopment analysis," Management Science, vol. 30, no. 9, pp. 1078-1092, 1984.

[4] Y. J. Lee, S.-J. Joo, and H. G. Park, "An application of data envelopment analysis for Korean banks with negative data," Benchmarking: An International Journal, vol. 24, no. 4, pp. 1052-1064, 2017.

[5] S. Kaffash, R. Azizi, Y. Huang, and J. Zhu, "A survey of data envelopment analysis applications in the insurance industry 1993-2018," European Journal of Operational Research, vol. 284, no. 3, pp. 801-813, 2020.

[6] H. H. Liu, J. J. Huang, and Y. H. Chiu, "Integration of network data envelopment analysis and decision-making trial and evaluation laboratory for the performance evaluation of the financial holding companies in Taiwan," Managerial and Decision Economics, vol. 41, no. 1, pp. 64-78, 2020.

[7] L. A. Moncayo-Martínez, A. Ramírez-Nafarrate, and M. G. Hernández-Balderrama, "Evaluation of public HEI on teaching, research, and knowledge dissemination by Data Envelopment Analysis," Socio-Economic Planning Sciences, vol. 69, p. 100718, 2020.

[8] N. Ai, M. Kjerland, C. Klein-Banai, and T. L. Theis, "Sustainability assessment of universities as small-scale urban systems: a comparative analysis using Fisher Information and Data Envelopment Analysis," Journal of Cleaner Production, vol. 212, pp. 1357-1367, 2019.

[9] D. Fernández, C. Pozo, R. Folgado, L. Jiménez, and G. Guillén-Gosálbez, "Productivity and energy efficiency assessment of existing industrial gases facilities via data envelopment analysis and the Malmquist index," Applied Energy, vol. 212, pp. 1563-1577, 2018.

[10] S. Kohl, J. Schoenfelder, A. Fügener, and J. O. Brunner, "The use of Data Envelopment Analysis (DEA) in healthcare with a focus on hospitals," Health Care Management Science, vol. 22, no. 2, pp. 245-286, 2019.

[11] M. Shafiee, F. Hosseinzadeh Lotfi, and H. Saleh, "Supply chain performance evaluation with data envelopment analysis and balanced scorecard approach," Applied Mathematical Modelling, vol. 38, no. 21-22, pp. 5092-5112, 2014.

[12] A. Emrouznejad and G.-l. Yang, "A survey and analysis of the first 40 years of scholarly literature in DEA: 1978-2016," SocioEconomic Planning Sciences, vol. 61, pp. 4-8, 2018.

[13] H. Chen, J. Liu, Y. Li, Y.-H. Chiu, and T.-Y. Lin, "A two-stage dynamic undesirable data envelopment analysis model focused on media reports and the impact on energy and health efficiency," International Journal of Environmental Research and Public Health, vol. 16, no. 9, p. 1535, 2019.

[14] S. Soheilirad, K. Govindan, A. Mardani, E. K. Zavadskas, M. Nilashi, and N. Zakuan, "Application of data envelopment analysis models in supply chain management: a systematic review and meta-analysis," Annals of Operations Research, vol. 271, no. 2, pp. 915-969, 2018.

[15] R. Lacko, Z. Hajduová, and V. Gábor, "Data envelopment analysis of selected specialized health centres and possibilities of its application in the terms of Slovak republic health care 
system," Journal of Health Management, vol. 19, no. 1, pp. 144-158, 2017.

[16] E. Krmac and B. Djordjević, "A new DEA model for evaluation of supply chains: a case of selection and evaluation of environmental efficiency of suppliers," Symmetry, vol. 11, no. 4, 2019.

[17] A. Hatami-Marbini, A. Emrouznejad, and M. Tavana, "A taxonomy and review of the fuzzy data envelopment analysis literature: two decades in the making," European Journal of Operational Research, vol. 214, no. 3, pp. 457-472, 2011.

[18] A. Emrouznejad, M. Tavana, and A. Hatami-Marbini, "The state of the art in fuzzy data envelopment analysis," Performance Measurement with Fuzzy Data Envelopment Analysis, vol. 309, pp. 1-45, 2014.

[19] F. Hosseinzadeh Lotfi, A. Ebrahimnejad, M. Vaez-Ghasemi, and Z. Moghaddas, Data Envelopment Analysis with $R$, Springer International Publishing, Cham, Switzerland, 2020.

[20] K. T. Atanassov, "Intuitionistic fuzzy sets," Fuzzy Sets and Systems, vol. 20, no. 1, pp. 87-96, 1986.

[21] R. R. Yager, "Pythagorean fuzzy subsets," in Proceedings of the 2013 Joint IFSA World Congress and NAFIPS Annual Meeting, pp. 57-61, IFSA/NAFIPS, Edmonton, Canada, 2013.

[22] L. Wang and N. Li, "Pythagorean fuzzy interaction power Bonferroni mean aggregation operators in multiple attribute decision making," International Journal of Intelligent Systems, vol. 35, no. 1, pp. 150-183, 2020.

[23] R. Kumar, S. A. Edalatpanah, S. Jha, and R. Singh, "A Pythagorean fuzzy approach to the transportation problem," Complex \& Intelligent Systems, vol. 5, no. 2, pp. 255-263, 2019.

[24] F. Smarandache, A Unifying Field in Logics. Neutrosophy: Neutrosophic Probability, Set and Logic, American Research Press, Rehoboth, MA, USA, 1999.

[25] F. G. Lupiáñez, "Interval neutrosophic sets and topology," Kybernetes.vol. 38, no. 3-4, pp. 621-624, 2009.

[26] H. Garg and Nancy, "Multiple attribute decision making based on immediate probabilities aggregation operators for single-valued and interval neutrosophic sets," Journal of Applied Mathematics and Computing, vol. 63, no. 1-2, pp. 619-653, 2020.

[27] Y. Yuan, Y. Ren, Y. Ren, X. Liu, and J. Wang, "Approach to image segmentation based on interval neutrosophic set," Numerical Algebra, Control \& Optimization, vol. 10, no. 1, pp. 1-11, 2020.

[28] S. Broumi, A. Bakali, M. Talea, F. Smarandache, and M. Ali, "Shortest path problem under bipolar neutrosphic setting," Applied Mechanics and Materials, vol. 859, pp. 59-66, 2016.

[29] V. Uluçay, I. Deli, and M. Şahin, "Similarity measures of bipolar neutrosophic sets and their application to multiple criteria decision making," Neural Computing and Applications, vol. 29, no. 3, pp. 739-748, 2018.

[30] J. Ye, "Multicriteria decision-making method using the correlation coefficient under single-valued neutrosophic environment," International Journal of General Systems, vol. 42, no. 4, pp. 386-394, 2013.

[31] S. Broumi, F. Smarandache, M. Talea, and A. Bakali, "Single valued neutrosophic graphs: degree, order and size," in Proceedings of the 2016 IEEE International Conference on Fuzzy Systems, pp. 2444-2451, FUZZ-IEEE, New Orleans, LA, USA, 2016.

[32] X. Peng and J. Dai, "Approaches to single-valued neutrosophic MADM based on MABAC, TOPSIS and new similarity measure with score function," Neural Computing and Applications, vol. 29, no. 10, pp. 939-954, 2018.
[33] H. Garg, "Novel neutrality aggregation operator-based multiattribute group decision-making method for single-valued neutrosophic numbers," Soft Computing, vol. 24, no. 14., pp. 10327-10349, 2020

[34] J. Ye, "A multicriteria decision-making method using aggregation operators for simplified neutrosophic sets," Journal of Intelligent \& Fuzzy Systems, vol. 26, no. 5, pp. 2459-2466, 2014.

[35] J.-J. Peng, J.-Q. Wang, H.-Y. Zhang, and X.-H. Chen, “An outranking approach for multi-criteria decision-making problems with simplified neutrosophic sets," Applied Soft Computing, vol. 25, pp. 336-346, 2014.

[36] J. Ye, "Generalized ordered weighted simplified neutrosophic cosine similarity measure for multiple attribute group decision making," International Journal of Cognitive Informatics and Natural Intelligence, vol. 14, no. 1, pp. 51-62, 2020.

[37] S. A. Edalatpanah, "Neutrosophic structured element," Expert Systems., Article ID e12542, 2020.

[38] J.-J. Peng, J.-Q. Wang, X.-H. Wu, J. Wang, and X.-H. Chen, "Multi-valued neutrosophic sets and power aggregation operators with their applications in multi-criteria group decision-making problems," International Journal of Computational Intelligence Systems, vol. 8, no. 2, pp. 345-363, 2015.

[39] N. L. A. M. Kamal, L. Abdullah, I. Abdullah, S. Alkhazaleh, and F. Karaaslan, "Multi-valued interval neutrosophic soft set: formulation and theory," Neutrosophic Sets and Systems, vol. 30, pp. 149-170, 2019.

[40] J. Ye, "Some aggregation operators of interval neutrosophic linguistic numbers for multiple attribute decision making," Journal of Intelligent \& Fuzzy Systems, vol. 27, no. 5, pp. 2231-2241, 2014.

[41] J. Ye, "Hesitant interval neutrosophic linguistic set and its application in multiple attribute decision making," International Journal of Machine Learning and Cybernetics, vol. 10, no. 4, pp. 667-678, 2019.

[42] S. A. Edalatpanah, "Systems of neutrosophic linear equations," Neutrosophic Sets and Systems, vol. 33, pp. 92-104, 2020.

[43] S. A. Edalatpanah, "A nonlinear approach for neutrosophic linear programming," Journal of Applied Research on Industrial Engineering, vol. 6, no. 4, pp. 367-373, 2019.

[44] S. K. Das and S. A. Edalatpanah, "A new ranking function of triangular neutrosophic number and its application in integer programming," International Journal of Neutrosophic Science, vol. 4, no. 2, pp. 82-92, 2020.

[45] S. A. Edalatpanah, "A direct model for triangular neutrosophic linear programming," International Journal of Neutrosophic Science, vol. 1, no. 1, pp. 19-28, 2020.

[46] R. Kumar, S. A. Edaltpanah, S. Jha, S. Broumi, and A. Dey, "Neutrosophic shortest path problem," Neutrosophic Sets and Systems, vol. 23, pp. 5-15, 2018.

[47] H. Garg, "Algorithms for single-valued neutrosophic decision making based on topsis and clustering methods with new distance measure," AIMS Mathematics, vol. 5, no. 3, pp. 2671-2693, 2020.

[48] U. Rivieccio, "Neutrosophic logics: prospects and problems," Fuzzy Sets and Systems, vol. 159, no. 14, pp. 1860-1868, 2008.

[49] X. Peng and J. Dai, "A bibliometric analysis of neutrosophic set: two decades review from 1998 to 2017," Artificial Intelligence Review, vol. 53, no. 1, pp. 199-255, 2020.

[50] G. N. Nguyen, L. H. Son, A. S. Ashour, and N. Dey, "A survey of the state-of-the-arts on neutrosophic sets in biomedical 
diagnoses," International Journal of Machine Learning and Cybernetics, vol. 10, no. 1, 2019.

[51] M. Abdel-Basset, G. Manogaran, A. Gamal, and F. Smarandache, "A hybrid approach of neutrosophic sets and DEMATEL method for developing supplier selection criteria," Design Automation for Embedded Systems, vol. 22, no. 3, pp. 257-278, 2018.

[52] S. A. Edalatpanah, "A data envelopment analysis model with triangular intuitionistic fuzzy numbers," International Journal of Data Envelopment Analysis, vol. 7, no. 4, pp. 47-58, 2019.

[53] A. Arya and S. P. Yadav, "A new approach to rank the decision making units in presence of infeasibility in intuitionistic fuzzy environment," Iranian Journal of Fuzzy Systems, vol. 17, no. 2, pp. 183-199, 2020.

[54] J. Puri and S. P. Yadav, "Intuitionistic fuzzy data envelopment analysis: an application to the banking sector in India," Expert Systems with Applications, vol. 42, no. 11, pp. 4982-4998, 2015.

[55] B. D. Rouyendegh, A. Yildizbasi, and I. Yilmaz, "Evaluation of retail industry performance ability through integrated intuitionistic fuzzy TOPSIS and data envelopment analysis approach," Soft Computing, vol. 24, no. 16, pp. 12255-12266, 2020.

[56] W. Zhou, J. Chen, B. Ding, and S. Meng, "Interval-valued intuitionistic fuzzy envelopment analysis and preference fusion," Computers \& Industrial Engineering, vol. 142, p. 106361, 2020.

[57] J. Liu, J. Song, Q. Xu, Z. Tao, and H. Chen, “Group decision making based on DEA cross-efficiency with intuitionistic fuzzy preference relations," Fuzzy Optimization and Decision Making, vol. 18, no. 3, pp. 345-370, 2019.

[58] A. Arya and S. P. Yadav, "Development of intuitionistic fuzzy data envelopment analysis models and intuitionistic fuzzy input-output targets," Soft Computing, vol. 23, no. 18, pp. 8975-8993, 2019.

[59] S. Singh, "Intuitionistic fuzzy dea/ar and its application to flexible manufacturing systems," RAIRO-Operations Research, vol. 52, no. 1, pp. 241-257, 2018.

[60] S. H. R. Hajiagha, H. Akrami, E. Kazimieras Zavadskas, and S. S. Hashemi, "An intuitionistic fuzzy data envelopment analysis for efficiency evaluation under uncertainty: case of a finance and credit institution," E a M: Ekonomie a Management, vol. 161, pp. 128-137, 2013.

[61] S. A. Edalatpanah, "Neutrosophic perspective on DEA," Journal of Applied Research on Industrial Engineering, vol. 5, no. 4, pp. 339-345, 2018.

[62] W. Yang, L. Cai, S. A. Edalatpanah, and F. Smarandache, "Triangular single valued neutrosophic data envelopment analysis: application to hospital performance measurement," Symmetry, vol. 12, no. 4, p. 588, 2020.

[63] W. Abdelfattah, "Data envelopment analysis with neutrosophic inputs and outputs," Expert Systems.vol. 36, no. 6, Article ID e12453, 2019.

[64] C. Kahraman, İ. Otay, B. Öztay, and S. Ç. Onar, "An integrated AHP \& DEA methodology with neutrosophic sets," in Studies in Fuzziness and Soft Computing, pp. 623-645, Springer-Verlag, Berlin Germany, 2019.

[65] S. A. Edalatpanah, "Data envelopment analysis based on triangular neutrosophic numbers," CAAI Transactions on Intelligence Technology, vol. 5, no. 2, pp. 94-98, 2020.

[66] S. A. Edalatpanah and F. Smarandache, "Data envelopment analysis for simplified neutrosophic Sets," vol. 29, 2019.
[67] N. Nabeeh, "A hybrid neutrosophic approach of DEMATEL with AR-DEA in technology selection," Neutrosophic Sets and Systems, vol. 31, pp. 17-30, 2020.

[68] D. Guo and J. Wu, "A complete ranking of DMUs with undesirable outputs using restrictions in DEA models," Mathematical and Computer Modelling, vol. 58, no. 5-6, pp. 1102-1109, 2013.

[69] R. Färe and S. Grosskopf, "Modeling undesirable factors in efficiency evaluation: comment.,", European Journal of Operational Research, vol. 157, no. 1, pp. 242-245, 2004.

[70] B. Walheer, "Output, input, and undesirable output interconnections in data envelopment analysis: convexity and returns-to-scale," Annals of Operations Research, vol. 284, no. 1, pp. 447-467, 2020.

[71] S. Lozano, E. Gutiérrez, and P. Moreno, "Network DEA approach to airports performance assessment considering undesirable outputs," Applied Mathematical Modelling, vol. 37, no. 4, pp. 1665-1676, 2013.

[72] A. Hadi Vencheh, R. Kazemi Matin, and M. Tavassoli Kajani, "Undesirable factors in efficiency measurement," Applied Mathematics and Computation, vol. 163, no. 2, pp. 547-552, 2005.

[73] W. Zhu, M. Xu, and C.-P. Cheng, "Dealing with undesirable outputs in DEA: an aggregation method for a common set of weights," Journal of the Operational Research Society, vol. 71, no. 4 , pp. $579-588,2020$. 